\title{
Specific Phospholipid Modulation by Muscarinic Signaling in a Rat Lesion Model of Alzheimer's Disease
}

Alberto Llorente-Ovejero ${ }^{1}$, Jonatan Martínez-Gardeazabal'1, Marta Moreno-Rodríguez ${ }^{1}$, Laura Lombardero ${ }^{1}$, Estíbaliz González de San Román ${ }^{1}$, Iván Manuel ${ }^{1,2}$, María Teresa Giralt ${ }^{1}$, Rafael Rodríguez-Puertas ${ }^{1,2}$ *

${ }^{1}$ Department of Pharmacology, Faculty of Medicine and Nursing. University of the Basque Country (UPV/EHU), $B^{\circ}$ Sarriena $s / n, 48940$ Leioa, Spain.

${ }^{2}$ Neurodegenerative Diseases, BioCruces Bizkaia Health Research Institute, Barakaldo, Spain. 
Table S1. Relative intensity of representative lipid species which have been found to be modified in the NBM and cortex of vehicle (aCSF) and 192lgG-saporin treated rats, in both positive and negative ion mode.

\begin{tabular}{|c|c|c|c|c|c|c|}
\hline \multirow[b]{3}{*}{ Assignment } & \multirow[b]{3}{*}{ Cal m/z } & \multirow[b]{3}{*}{ Exp $\mathrm{m} / \mathrm{z}$} & & \multicolumn{3}{|c|}{$\%$ Intensity ${ }^{[1]}$} \\
\hline & & & & NBM & \multicolumn{2}{|c|}{ Cortex } \\
\hline & & & aCSF & SAP & aCSF & SAP \\
\hline \multicolumn{7}{|l|}{ Positive ion mode } \\
\hline $\operatorname{PE}(14: 1 / 20: 4)+\mathrm{H}^{+}$ & 710.4755 & 710.4893 & $15.21 \pm 0.70$ & $11.87 \pm 1.38^{*}$ & $9.97 \pm 0.62$ & $10.67 \pm 0.67$ \\
\hline$S M(d 18: 1 / 18: 0)+H^{+}$ & 731.6061 & 731.6070 & $7.11 \pm 1.13$ & $4.15 \pm 0.80^{*}$ & $4.19 \pm 0.44$ & $3.50 \pm 0.53$ \\
\hline$S M(d 18: 1 / 16: 0)+K^{+}$ & 741.5313 & 741.5311 & $0.96 \pm 0.14$ & $10.63 \pm 3.33^{* *}$ & $0.55 \pm 0.13$ & $0.78 \pm 0.11$ \\
\hline $\mathrm{PC}(32: 0)+\mathrm{Na}^{+} / \mathrm{PC}(34: 3)+\mathrm{H}^{+}$ & 756.5514 & 756.5520 & $15.75 \pm 1.23$ & $21.94 \pm 2.46^{*}$ & $16.94 \pm 1.02$ & $17.41 \pm 1.31$ \\
\hline $\mathrm{PC}(34: 2)+\mathrm{Na}^{+} / \mathrm{PC}(36: 5)+\mathrm{H}^{+}$ & 780.5514 & 780.5521 & $0.85 \pm 0.09$ & $2.02 \pm 0.27^{\star *}$ & $1.10 \pm 0.08$ & $1.07 \pm 0.08$ \\
\hline $\mathrm{PC}(34: 1)+\mathrm{Na}^{+} / \mathrm{PC}(36: 4)+\mathrm{H}^{+}$ & 782.5670 & 782.5673 & $32.75 \pm 1.65$ & $46.10 \pm 3.86^{* *}$ & $32.31 \pm 1.64$ & $30.9 \pm 1.29$ \\
\hline $\mathrm{PC}(34: 2)+\mathrm{K}^{+}$ & 796.5253 & 796.5255 & $3.27 \pm 0.17$ & $5.2 \pm 0.30^{\star * *}$ & $4.20 \pm 0.15$ & $4.16 \pm 0.07$ \\
\hline$P C(34: 1)+K^{+}$ & 798.5410 & 798.5405 & $100 \pm 0$ & $100 \pm 0$ & $100 \pm 0$ & $100 \pm 0$ \\
\hline $\mathrm{PC}(36: 4)+\mathrm{Na}^{+} / \mathrm{PC}(38: 7)+\mathrm{H}^{+}$ & 804.5514 & 804.5510 & $2.79 \pm 0.31$ & $7.69 \pm 1.52^{*}$ & $3.63 \pm 0.31$ & $4.3 \pm 0.24$ \\
\hline $\mathrm{PC}(36: 1)+\mathrm{Na}^{+} / \mathrm{PC}(38: 4)+\mathrm{H}^{+}$ & 810.5983 & 810.5984 & $14.62 \pm 0.30$ & $21.64 \pm 1.53^{\star *}$ & $10.62 \pm 0.48$ & $8.95 \pm 0.63^{*}$ \\
\hline$P C(36: 4)+K^{+}$ & 820.5253 & 820.5249 & $10.32 \pm 1.01$ & $18.61 \pm 2.71^{*}$ & $13.17 \pm 0.99$ & $15.63 \pm 0.48^{*}$ \\
\hline $\mathrm{PC}(38: 4)+\mathrm{Na}^{+} / \mathrm{PC}(40: 7)+\mathrm{H}^{+}$ & 832.5827 & 832.5826 & $3.00 \pm 0.30$ & $7.98 \pm 1.40^{\star \star}$ & $2.82 \pm 0.25$ & $3.11 \pm 0.21$ \\
\hline $\mathrm{PC}(38: 4)+\mathrm{K}^{+} / \mathrm{PC}(42: 9)+\mathrm{H}^{+}$ & 848.5566 & 848.5560 & $10.23 \pm 0.93$ & $18.27 \pm 2.15^{\star \star}$ & $9.71 \pm 0.80$ & $10.75 \pm 0.38$ \\
\hline $\mathrm{PC}(40: 6)+\mathrm{Na}^{+}$ & 856.5827 & 856.5820 & $0.88 \pm 0.17$ & $2.25 \pm 0.40^{* *}$ & $0.75 \pm 0.11$ & $1.14 \pm 0.09^{*}$ \\
\hline \multicolumn{7}{|l|}{ Negative ion mode } \\
\hline $\mathrm{SM}(\mathrm{d} 18: 1 / 15: 0)-\mathrm{H}^{-}$ & 687.5447 & 687.5443 & $1.26 \pm 0.24$ & $6.44 \pm 1.50^{* *}$ & $0.94 \pm 0.23$ & $1.52 \pm 0.28$ \\
\hline PS (18:0/18:1) - $\mathrm{H}^{-}$ & 788.5447 & 788.5447 & $11.04 \pm 2.16$ & $3.95 \pm 0.99^{* *}$ & $8.54 \pm 1.28$ & $6.30 \pm 0.85$ \\
\hline $\mathrm{PE}(40: 4) / \mathrm{PC}(18: 0 / 20: 4)-\mathrm{H}^{-}$ & 794.5705 & 794.5705 & $0.82 \pm 0.13$ & $1.35 \pm 0.17^{*}$ & $0.83 \pm 0.08$ & $0.98 \pm 0.14$ \\
\hline PG $(22: 6 / 22: 6)-\mathrm{H}^{-}$ & 865.5025 & 865.5033 & $0.06 \pm 0.01$ & $0.86 \pm 0.24^{\star \star}$ & $0.04 \pm 0.01$ & $0.16 \pm 0.05^{*}$ \\
\hline
\end{tabular}

[1] The maximal peak is the most intense peak of the lipid spectrum, in this case PC $(34: 1)$ in positive and $\mathrm{PI}(20: 4 / 18: 0)$ in negative ion modes, which are set at $100 \%$. Data are mean \pm S.E.M values of aCSF $(n=8)$ and 192lgG-saporin-treated $(n=8)$ rats. ${ }^{*} p<0.05,{ }^{* *} p<0.01$ and ${ }^{* * *} p<0.001$ when compared to aCSF group. PC: phosphatidylcholine; SM: sphingomyelin; PS: phosphatidylserine; PE: phosphatidylethanolamine; PG: phosphoglycerol; PI: phosphatidylinositol; Cal: calculated; Exp: experimental. 
Table S2. ${ }^{35}$ S]GTP $\gamma$ S basal and carbachol-induced $(100 \mu \mathrm{M})$ binding in cortical, hippocampal and several brain regions of vehicle (aCSF) and 192lgG-saporin-treated rats.

\begin{tabular}{|c|c|c|c|c|}
\hline \multirow[b]{2}{*}{ Brain region } & \multicolumn{2}{|c|}{$\begin{array}{l}\text { Basal binding } \\
\text { (nCi/g t.e.) }\end{array}$} & \multicolumn{2}{|c|}{$\begin{array}{l}\text { Carbachol stimulation } \\
\text { (\% Over basal) }\end{array}$} \\
\hline & aCSF & SAP & aCSF & SAP \\
\hline \multicolumn{5}{|l|}{ Cerebral cortex } \\
\hline Cingulate & $304 \pm 26$ & $306 \pm 20$ & $52 \pm 12.1$ & $48 \pm 6.7$ \\
\hline Ectorhinal & $352 \pm 34$ & $290 \pm 29$ & $66 \pm 12.5$ & $58 \pm 8.0$ \\
\hline Entorhinal & $342 \pm 35$ & $335 \pm 36$ & $76 \pm 10.5$ & $93 \pm 11.6$ \\
\hline Perirhinal & $338 \pm 49$ & $309 \pm 29$ & $35 \pm 5.8$ & $43 \pm 3.5$ \\
\hline Somatosensory & $355 \pm 28$ & $333 \pm 20$ & $31 \pm 7.0$ & $54 \pm 10.6$ \\
\hline Motor & $311 \pm 33$ & $319 \pm 20$ & $40 \pm 13.3$ & $66 \pm 6.5$ \\
\hline \multicolumn{5}{|l|}{ Hippocampus } \\
\hline \multicolumn{5}{|l|}{ CA1 } \\
\hline Oriens & $320 \pm 40$ & $269 \pm 32$ & $26 \pm 9.4$ & $40 \pm 10.4$ \\
\hline Pyramidal & $527 \pm 64$ & $457 \pm 50$ & $40 \pm 11.5$ & $30 \pm 6.2$ \\
\hline Radiatum & $363 \pm 33$ & $323 \pm 36$ & $45 \pm 11.5$ & $42 \pm 9.0$ \\
\hline \multicolumn{5}{|l|}{ CA3 } \\
\hline Oriens & $317 \pm 26$ & $303 \pm 38$ & $26 \pm 8.0$ & $48 \pm 6.6$ \\
\hline Pyramidal & $477 \pm 36$ & $453 \pm 42$ & $11 \pm 5.6$ & $32 \pm 4.2^{*}$ \\
\hline Radiatum & $328 \pm 21$ & $281 \pm 32$ & $28 \pm 9.5$ & $40 \pm 13.0$ \\
\hline \multicolumn{5}{|l|}{ Dentate gyrus } \\
\hline Granular & $467 \pm 29$ & $452 \pm 50$ & $24 \pm 6.1$ & $48 \pm 9.7^{*}$ \\
\hline Molecular & $315 \pm 49$ & $278 \pm 45$ & $34 \pm 14.7$ & $34 \pm 9.5$ \\
\hline \multicolumn{5}{|l|}{ Mesencephalon } \\
\hline Periaqueductal gray & $496 \pm 44$ & $469 \pm 45$ & $51 \pm 15.0$ & $47 \pm 8.5$ \\
\hline Substantia nigra & $456 \pm 52$ & $399 \pm 38$ & $31 \pm 10.5$ & $37 \pm 5.4$ \\
\hline \multicolumn{5}{|c|}{ Basal ganglia/cholinergic forebrain } \\
\hline Globus pallidus & $335 \pm 43$ & $347 \pm 36$ & $46 \pm 13.2$ & $49 \pm 12.5$ \\
\hline Striatum & $339 \pm 26$ & $345 \pm 21$ & $74 \pm 15.4$ & $62 \pm 11.1$ \\
\hline NBM & $535 \pm 49$ & $398 \pm 31$ & $43 \pm 9.2$ & $11 \pm 7.5^{*}$ \\
\hline Horiz. diagonal band & $310 \pm 25$ & $327 \pm 31$ & $121 \pm 20.7$ & $115 \pm 17.6$ \\
\hline Vertical diagonal band & $330 \pm 25$ & $399 \pm 42$ & $135 \pm 24.1$ & $113 \pm 23.2$ \\
\hline Medial septum & $267 \pm 24$ & $284 \pm 32$ & $152 \pm 24.4$ & $139 \pm 23.7$ \\
\hline
\end{tabular}

Data are mean \pm S.E. M values of aCSF $(n=9)$ and SAP $(n=11)$ treated rats.

${ }^{*} p<0.05$, when compared to aCSF group. 
Table S3. ${ }^{35}$ S]GTP $\gamma$ S basal and carbachol-induced $(100 \mu \mathrm{M})$ binding in the different amygdaloid nuclei and several brain regions of vehicle (aCSF) and 192lgG-saporin-treated rats.

\begin{tabular}{|c|c|c|c|c|}
\hline \multirow[b]{2}{*}{ Brain region } & \multicolumn{2}{|c|}{$\begin{array}{c}\text { Basal binding } \\
\text { (nCi/g t.e.) }\end{array}$} & \multicolumn{2}{|c|}{$\begin{array}{c}\text { Carbachol stimulation } \\
(\% \text { Over basal) }\end{array}$} \\
\hline & aCSF & SAP & aCSF & SAP \\
\hline \multicolumn{5}{|l|}{ Telencephalon } \\
\hline \multicolumn{5}{|l|}{ Amygdaloid nuclei } \\
\hline Anterior & $421 \pm 33$ & $402 \pm 29$ & $59 \pm 9.1$ & $47 \pm 13.6$ \\
\hline Basolateral & $487 \pm 47$ & $390 \pm 44$ & $43 \pm 6.6$ & $48 \pm 10.9$ \\
\hline Central & $710 \pm 91$ & $525 \pm 55$ & $27 \pm 9.2$ & $52 \pm 7.8$ \\
\hline Lateral & $483 \pm 50$ & $425 \pm 43$ & $41 \pm 9.3$ & $36 \pm 7.2$ \\
\hline Medial & $720 \pm 94$ & $621 \pm 69$ & $39 \pm 8.5$ & $34 \pm 4.7$ \\
\hline Ventral subiculum & $345 \pm 29$ & $294 \pm 18$ & $37 \pm 4.8$ & $47 \pm 6.7$ \\
\hline \multicolumn{5}{|l|}{ Rhinencephalon } \\
\hline Lat olfactory tract & $379 \pm 45$ & $324 \pm 37$ & $82 \pm 13.1$ & $47 \pm 11.5$ \\
\hline \multicolumn{5}{|l|}{ Midbrain } \\
\hline AV thalamic nucleus & $425 \pm 32$ & $397 \pm 41$ & $232 \pm 17.4$ & $251 \pm 28.6$ \\
\hline Gray sup colliculus & $341 \pm 27$ & $362 \pm 36$ & $197 \pm 15.4$ & $211 \pm 19.9$ \\
\hline \multicolumn{5}{|l|}{ Rhomboencephalon } \\
\hline Dorsal raphe & $703 \pm 103$ & $590 \pm 74$ & $19 \pm 4.9$ & $24 \pm 4.7$ \\
\hline Locus coeruleus & $220 \pm 36$ & $132 \pm 16$ & $26 \pm 11.9$ & $78 \pm 11.9$ \\
\hline
\end{tabular}

Data are mean \pm S.E. M values of aCSF $(n=9)$ and SAP $(n=11)$ treated rats. 
Table S4. ${ }^{35}$ S] GTP $\gamma$ S basal and carbachol-induced $(100 \mu \mathrm{M})$ binding in different regions of grey matter from SHAM-operated rats.

\begin{tabular}{|c|c|c|c|c|c|}
\hline & $\begin{array}{c}\text { Basal } \\
\text { binding } \\
\text { (nCi/g t.e.) }\end{array}$ & $\begin{array}{c}\text { Carbachol } \\
\text { stimulation } \\
\text { (\% Over basal) }\end{array}$ & & $\begin{array}{c}\text { Basal } \\
\text { binding } \\
\text { (nCi/g t.e.) }\end{array}$ & $\begin{array}{c}\text { Carbachol } \\
\text { stimulation } \\
\text { (\% Over basal) }\end{array}$ \\
\hline Brain region & & & Brain region & & \\
\hline Telencephalon & & & Cerebral cortex & & \\
\hline Amygdala & & & Cingulate & $354 \pm 30$ & $43 \pm 12.7$ \\
\hline Anterior & $403 \pm 33$ & $37 \pm 14.4$ & Ectorhinal & $337 \pm 56$ & $36 \pm 6.5$ \\
\hline Basolateral & $387 \pm 41$ & $53 \pm 10.5$ & Entorhinal & $308 \pm 30$ & $47 \pm 7.1$ \\
\hline Central & $536 \pm 53$ & $32 \pm 8.39$ & Perirhinal & $309 \pm 43$ & $36 \pm 13.5$ \\
\hline Lateral & $373 \pm 53$ & $78 \pm 16.9$ & Piriform & $270 \pm 31$ & $61 \pm 9.8$ \\
\hline Medial & $672 \pm 84$ & $34 \pm 11.7$ & Somatosensory & $368 \pm 28$ & $34 \pm 4.9$ \\
\hline Hippocampus & & & Motor & $353 \pm 37$ & $34 \pm 14.8$ \\
\hline CA1 & & & Basal ganglia & & \\
\hline Oriens & $252 \pm 25$ & $27 \pm 12.0$ & Globus pallidus & $275 \pm 25$ & $54 \pm 12.2$ \\
\hline Pyramidal & $366 \pm 44$ & $55 \pm 15.6$ & Striatum & $330 \pm 37$ & $85 \pm 14.0$ \\
\hline Radiatum & $279 \pm 19$ & $42 \pm 9.8$ & Diencephalon & & \\
\hline CA3 & & & NBM & $492 \pm 51$ & $47 \pm 12.3$ \\
\hline Oriens & $280 \pm 44$ & $21 \pm 6.8$ & Horiz diag band & $356 \pm 29$ & $115 \pm 19.5$ \\
\hline Pyramidal & $419 \pm 52$ & $9 \pm 6.2$ & Vertical diag band & $366 \pm 28$ & $112 \pm 25.6$ \\
\hline Radiatum & $264 \pm 38$ & $40 \pm 19.8$ & Medial septum & $280 \pm 50$ & $150 \pm 41.2$ \\
\hline Dentate gyrus & & & Rhinencephalon & & \\
\hline Granular & $437 \pm 32$ & $25 \pm 1.2$ & Lat olfactory tract & $356 \pm 27$ & $41 \pm 9.8$ \\
\hline Molecular & $263 \pm 45$ & $44 \pm 9.2$ & Rhomboencephalon & & \\
\hline Polimorphic & $290 \pm 39$ & $28 \pm 15.7$ & Dorsal raphe & $533 \pm 99$ & $42 \pm 9.4$ \\
\hline Vent subic & $301 \pm 35$ & $49 \pm 11.4$ & Locus coeruleus & $197 \pm 30$ & $52 \pm 6.0$ \\
\hline Brainstem & & & Mesencephalon & & \\
\hline \multirow[t]{2}{*}{ Spinal trig $N$} & $178 \pm 25$ & $87 \pm 19.8$ & Periaqueduc gray & $450 \pm 82$ & $47 \pm 10.3$ \\
\hline & & & Substantia nigra & $361 \pm 56$ & $32 \pm 14.9$ \\
\hline
\end{tabular}

Data are mean \pm S.E. M values of SHAM-operated $(n=6)$ rats. 
Table S5. $\left[{ }^{3} \mathrm{H}\right]$-pirenzepine and $\left[{ }^{3} \mathrm{H}\right]$-oxotremorine binding in different brain regions of vehicle (aCSF) and 192lgG-saporin-treated rats.

\begin{tabular}{|c|c|c|c|c|c|}
\hline \multicolumn{3}{|c|}{$\begin{array}{c}{\left[{ }^{3} \mathrm{H}\right]-p i r e n z e p i n e ~ b i n d i n g} \\
(\mathrm{fmol} / \mathrm{mg} \text { t.e. })\end{array}$} & \multicolumn{3}{|c|}{$\begin{array}{c}{\left[{ }^{3} \mathrm{H}\right] \text {-oxotremorine binding }} \\
\text { (fmol/mg t.e.) }\end{array}$} \\
\hline Brain region & aCSF & SAP & Brain region & aCSF & SAP \\
\hline Amygdala & & & Amygdala & & \\
\hline Anterior & $7.3 \pm 0.8$ & $3.6 \pm 0.9^{*}$ & Anterior & $21.2 \pm 2.7$ & $31.1 \pm 1.9$ \\
\hline Basolateral & $70.2 \pm 5.9$ & $74.5 \pm 4.8$ & Basolateral & $5.2 \pm 1.7$ & $5.1 \pm 1.8$ \\
\hline Central & $30.5 \pm 3.6$ & $37.6 \pm 6.6$ & Lateral & $5.4 \pm 1.1$ & $5.3 \pm 1.6$ \\
\hline Lateral & $66.1 \pm 6.2$ & $66.1 \pm 4.8$ & Hippocampus & & \\
\hline Hippocampus & & & CA1 & & \\
\hline CA1 & $111.3 \pm 10$ & $131.8 \pm 8.8$ & Pyramidal & $6.2 \pm 1.5$ & $7.5 \pm 2.2$ \\
\hline Oriens & $114.8 \pm 10$ & $126.1 \pm 4.2$ & CA3 & & \\
\hline Pyramidal & $127.9 \pm 9.7$ & $134.9 \pm 7.9$ & Pyramidal & $11.0 \pm 2.3$ & $11.3 \pm 2.6$ \\
\hline Radiatum & $120.6 \pm 8.6$ & $136.2 \pm 7.7$ & Dentate gyrus & & \\
\hline CA3 & $58.2 \pm 6.6$ & $64.1 \pm 2.5$ & Granular & $3.6 \pm 1.5$ & $7.1 \pm 1.7$ \\
\hline Oriens & $43.4 \pm 3.4$ & $58.3 \pm 2.3^{* *}$ & Midbrain & & \\
\hline Pyramidal & $43.4 \pm 4.5$ & $53.5 \pm 4.1$ & AV thalamic nucleus & $66.3 \pm 4.4$ & $62.1 \pm 2.7$ \\
\hline Radiatum & $49.5 \pm 3.6$ & $58.2 \pm 3.6$ & Gray sup colliculus & $57.8 \pm 2.9$ & $72.9 \pm 4.5^{\star}$ \\
\hline Dentate gyrus & $108.1 \pm 4.5$ & $129.3 \pm 5.0^{*}$ & Opt sup colliculus & $28.8 \pm 5.3$ & $31.3 \pm 6.2$ \\
\hline Granular & $34.4 \pm 2.5$ & $46.4 \pm 2.0^{*}$ & Cerebral cortex & & \\
\hline Molecular & $106.3 \pm 8.8$ & $114.9 \pm 7.1$ & Cingulate & $11.2 \pm 2.3$ & $17.9 \pm 1.6^{*}$ \\
\hline Polimorphic & $74.9 \pm 5.5$ & $79.7 \pm 4.2$ & Ectorhinal & $10.0 \pm 2.1$ & $14.6 \pm 1.4$ \\
\hline Cerebral cortex & & & Perirhinal & $6.9 \pm 2.3$ & $10.7 \pm 1.2$ \\
\hline Cingulate & $38.2 \pm 1.0$ & $36.1 \pm 2.1$ & Motor & & \\
\hline Ectorhinal & $58.5 \pm 5.5$ & $54.6 \pm 2.9$ & Layer I-II & $19.6 \pm 2.6$ & $26.5 \pm 2.1$ \\
\hline Entorhinal & $51.4 \pm 4.6$ & $47.6 \pm 3.7$ & Layer V-VI & $12.7 \pm 3.0$ & $21.8 \pm 2.4^{*}$ \\
\hline Perirhinal & $55.2 \pm 4.7$ & $54.6 \pm 3.0$ & Somatosensory & & \\
\hline Motor & $39.5 \pm 3.1$ & $36.9 \pm 1.3$ & Layer I-II & $22.9 \pm 1.1$ & $29.7 \pm 2.5$ \\
\hline Somatosensory & $43.7 \pm 2.6$ & $43.9 \pm 2.6$ & Layer V-VI & $12.2 \pm 2.0$ & $20.3 \pm 2.5^{\star}$ \\
\hline \multicolumn{3}{|c|}{ Basal ganglia/cholinergic forebrain } & \multicolumn{3}{|c|}{ Basal ganglia/cholinergic forebrain } \\
\hline Striatum & $62.1 \pm 6.1$ & $63.8 \pm 3.2$ & Striatum & $8.2 \pm 1.9$ & $10.0 \pm 1.5$ \\
\hline $\begin{array}{l}\text { Globus pallidus } \\
\text { NBM }\end{array}$ & $\begin{array}{l}5.0 \pm 0.7 \\
5.4 \pm 1.4\end{array}$ & $\begin{array}{l}3.3 \pm 0.5 \\
4.5 \pm 0.7\end{array}$ & $\begin{array}{l}\text { Medial septum } \\
\text { NBM }\end{array}$ & $\begin{array}{r}30.1 \pm 1.7 \\
7.2 \pm 0.6\end{array}$ & $\begin{aligned} 31.2 & \pm 3.6 \\
3.1 & \pm 0.4^{\star \star *}\end{aligned}$ \\
\hline
\end{tabular}

Data are mean \pm S.E. M values of aCSF $(n=7)$ and SAP $(n=9)$ treated rats.

${ }^{*} p<0.05,{ }^{* *} p<0.01,{ }^{* * *} p<0.001$ when compared to aCSF group. 
Table S6. Absolute intensity of lipid species in organotypic cultures after treatments in both positive and negative ion mode.

\begin{tabular}{|c|c|c|c|c|c|c|c|c|c|c|c|c|c|c|}
\hline \multirow[b]{3}{*}{ Assignment } & \multirow[b]{3}{*}{ Cal $m / z$} & \multirow[b]{3}{*}{$\operatorname{Exp} m / z$} & \multirow{2}{*}{\multicolumn{12}{|c|}{ Intensity (u.a.) }} \\
\hline & & & & & & & & & & & & & & \\
\hline & & & \multicolumn{3}{|c|}{ VEHICLE } & \multicolumn{3}{|c|}{ CARBACHOL } & \multicolumn{3}{|c|}{ CAR+SCOP } & \multicolumn{3}{|c|}{ CAR+PIR } \\
\hline $\operatorname{LPC}(16: 0)+\mathrm{H}^{+}$ & 496.3398 & 496.3379 & $1.71 \cdot 10^{9}$ & \pm & 224629 & $4.7 \cdot 10^{9}$ & \pm & $6 \cdot 10^{5 * a}$ & $3.1 \cdot 10^{9}$ & \pm & $1 \cdot 10^{5}$ & $4.25 \mathrm{E}+09$ & \pm & $6 \cdot 10^{5 *} \mathrm{~b}$ \\
\hline $\operatorname{LPC}(16: 1)+\mathrm{H}^{+}$ & 494.3241 & 494.3270 & 3809. & \pm & 461 & 11696.00 & \pm & $2506^{*} a$ & 5400.00 & \pm & 1336 & 7250.00 & \pm & 2101 \\
\hline $\operatorname{LPC}(18: 0)+\mathrm{H}^{+}$ & 524.3744 & 524.3711 & 910345 & \pm & 166700 & $5.52 \cdot 10^{9}$ & \pm & $5 \cdot 10^{5 * a}$ & 4. $\cdot 10^{9}$ & \pm & $2 \cdot 10^{5}$ & $5.2 \cdot 10^{9}$ & \pm & $6 \cdot 10^{5 * b}$ \\
\hline $\operatorname{LPC}(0-18: 0)+\mathrm{H}^{+}$ & 508.3761 & 508.3788 & 6580 & \pm & 864 & 18654 & \pm & $2098^{* * a}$ & 10133 & \pm & 840 & 15956 & \pm & $1373^{*} \mathbf{b}$ \\
\hline LPC $(0-18: 1)+\mathrm{H}+$ & 506.3594 & 506.3605 & $8.84 \cdot 10^{4}$ & \pm & 13851 & $1.39 \cdot 10^{9}$ & \pm & $2 \cdot 10^{5 * a}$ & $9.5 \cdot 10^{5}$ & \pm & $1 \cdot 10^{5}$ & $1.4 \cdot 10^{9}$ & \pm & $1 \cdot 10^{5 * b}$ \\
\hline $\mathrm{PC}(36: 4)+\mathrm{H}^{+}$ & 782.5694 & 782.5727 & $9.29 \cdot 10^{9}$ & \pm & $1 \cdot 10^{9}$ & $5.89 \cdot 10^{9}$ & \pm & $3 \cdot 10^{5 * a}$ & $7.1 \cdot 10^{9}$ & \pm & $2 \cdot 10^{5}$ & $5.8 \cdot 10^{9}$ & \pm & $2 \cdot 10^{5 * * b}$ \\
\hline $\mathrm{PC}(\mathrm{O}-36: 4)+\mathrm{H}^{+}$ & 768.5902 & 768.5943 & 173714 & \pm & 7706 & 54424 & \pm & $9721^{* a}$ & 146795 & \pm & 6801 & 69580 & \pm & $12002^{*} b$ \\
\hline $\mathrm{PC}(36: 5)+\mathrm{Na}^{+}$ & 802.5357 & 802.5401 & 44361 & \pm & 1268 & 23056 & \pm & 3204 & 35767 & \pm & 6284 & 14701 & \pm & $3088^{* * b}$ \\
\hline $\mathrm{PC}(38: 5)+\mathrm{H}^{+}$ & 808.5851 & 808.5861 & 761567 & \pm & 42629 & 526654 & \pm & $21651^{* a}$ & 700222 & \pm & 63349 & 568492 & \pm & 48686 \\
\hline $\mathrm{PC}(38: 6)+\mathrm{H}^{+}$ & 806.5694 & 806.5742 & 764787 & \pm & 81976 & 349308 & \pm & $23788^{* * a}$ & 481258 & \pm & 32195 & 349138 & \pm & $4729 * b$ \\
\hline $\mathrm{PC}(38: 7)+\mathrm{H}^{+}$ & 804.5565 & 804.5538 & 597052 & \pm & 100617 & 270280 & \pm & $26512^{\star} a$ & 402153 & \pm & 59147 & 248105 & \pm & $40459 * b$ \\
\hline $\mathrm{PC}(\mathrm{O}-38: 7)+\mathrm{H}^{+}$ & 790.5745 & 790.5795 & 63971 & \pm & 5613 & 26141 & \pm & $4941^{* a}$ & 64351 & \pm & 9306 & 30403 & \pm & 4465 \\
\hline $\begin{array}{l}\mathrm{PC}(40: 7)+\mathrm{Na}^{+} / \mathrm{PC} \\
(36: 4)+\mathrm{H}^{+}\end{array}$ & 832.5851 & 832.5851 & 288228 & \pm & $24796^{*}$ & 197092 & \pm & 19490 & 282757 & \pm & 21872 & 185810 & \pm & 35558 \\
\hline \multicolumn{15}{|l|}{ Negative ion mode } \\
\hline LPC (O-18:0) $-\mathrm{CH}_{3}$ & 508.3409 & 508.3418 & 3997 & \pm & 1106 & 18226 & \pm & $1697^{*} \mathrm{a}$ & 11564 & \pm & 1505 & 20301 & \pm & $3041^{* *} b$ \\
\hline
\end{tabular}

The maximal peak in negative ion mode, $\mathrm{PI}(20: 4 / 18: 0)$, changed with treatments. Data are mean \pm S.E.M values of absolute intensity of VEHICLE $(n=6)$, CARBACHOL $(n=4)$, CAR+SCOP: Carbachol+Scopolamine $(n=4)$, and CAR+PIR: Carbachol+Pirenzepine $(n=4)$ of absolute intensity. ${ }^{*} p<0.05,{ }^{* *} p<0.01$. $\mathbf{a}^{*}$ when compared VEHICLE vs CARBACHOL. $\mathbf{b}^{*}$ when compared VEHICLE vs CAR+PYR. PC: phosphatidylcholine; LPC: phosphatidylcholine; Cal: calculated; Exp: experimental. 